\title{
Rendimiento y valor nutricional de tres variedades de sorgo dulce cultivadas en cuatro ambientes de Durango
}

\section{Yield and nutritional value of three sweet sorghum varieties grown at four environments in Durango}

\author{
Cynthia Adriana Nava Berumen ${ }^{a}$, Rigoberto Rosales Serna ${ }^{b}$, Rafael J iménez Ocampo ${ }^{b^{*}}$, Francisco Óscar \\ Carrete Carreónc, Pablo Alfredo Domínguez Martínez ${ }^{b}$, Manuel Murillo Ortiz ${ }^{c}$
}

\begin{abstract}
RESUMEN
El sorgo dulce muestra alto rendimiento de forraje para la alimentación de rumiantes. El objetivo fue evaluar el rendimiento y calidad forrajera de tres variedades de sorgo en Durango. En 2014, se evaluaron las variedades Lico, TOM 3 y Mercedes, en la Colonia Hidalgo (CH), Campo Experimental Valle del Guadiana (CEVAG), La Soledad (LS) y La Goma (LG). El diseño experimental fue completamente aleatorio con arreglo factorial y cinco repeticiones. Se evaluó forraje verde (FV), forraje seco (FS), proteína cruda (PC), digestibilidad verdadera in vitro (DI VMS), fibra detergente neutra (FDN), fibra detergente ácida (FDA), lignina (L), celulosa (Cel), hemicelulosa (HE), valor relativo (VRF) y calidad relativa del forraje (CRF). Se observaron diferencias significativas ( $P \leq 0.01$ y 0.05) entre ambientes y variedades para FV, FS, L, VRF y CRF y entre ambientes para PC, FDN, FDA, CEL, HE y DI VMS. En CEVAG, se registró el mayor rendimiento de FV (75.9 t ha-1) y FS (12.4 t ha-1). El valor más alto de PC se obtuvo en LG (10\% ); mientras que la DI VMS fue alta en LS (78.2 \%) y más baja en LG (63.9 \%). El contenido de L fue más alto en CH (6.3 \%) y LG (6.2 \%); mientras que, en LS fue de $3.4 \%$. Exceptuando LG, en todos los ambientes se obtuvo alta calidad forrajera (CRF=106.7 a $135.0 \%$ ). El sorgo es una opción forrajera en Durango, aunque debe cuidarse la fecha de siembra y cosecha, control de insectos, riego y fertilización para incrementar el rendimiento y calidad nutricional.
\end{abstract}

PALABRAS CLAVE: Sorghum bicolor, Composición química, Digestibilidad, Valor nutricional.

\section{ABSTRACT}

Sweet sorghum is a high-yielding biomass crop used as fodder for ruminant feed. The aim was to evaluate forage yield and quality in three sorghum cultivars grown in Durango. In 2014, three sorghum cultivars (Lico, TOM 3 and Mercedes) were sown in Colonia Hidalgo (CH), Valle del Guadiana Experiment Station (CEVAG), La Soledad (LS) and La Goma (LG). A completely randomized experimental design, with a factorial arrangement and five replications, was used. Data were taken for green (GF) and dry (DF) forage yield, crude protein (CP), in vitro dry matter true digestibility (IVTD), neutral detergent fiber (NDF), acid detergent fiber (ADF), lignin (L), cellulose (CEL), hemicellulose (HE), relative forage value (RFV) and relative forage quality (RFQ). Significant differences ( $P \leq 0.01$ and 0.05 ) among environments and cultivars were detected for $G F, D F, L, R F V$ and $R F Q$ and among environments for CP, NDF, ADF, CEL, HE and IVTD. The highest yield value for GF (75.9 t ha-1) and DF (12.4 t ha-1) were observed in CEVAG. Crude protein registered the highest value in LG (10\%); while IVTD showed the highest value in LS (78.2 \%) and the lowest in LG (63.9 $\%$ ). Lignin content was higher in CH (6.3\%) and LG (6.2 \% ); while in LS was $3.4 \%$. High quality fodder was obtained according to RFQ (106.7 to $135.0 \%$ ), except in LG. Sweet sorghum is an option as forage crop in Durango, however late plantings, early cutting dates (boot), insect control, irrigation and fertilizer application are recommended in order to increase its yield and nutritional quality.

KEY WORDS: Sorghum bicolor, Chemical composition, Digestibility, Nutritional value.

\footnotetext{
Recibido el 15 de diciembre de 2015. Aceptado el 28 de enero de 2016.

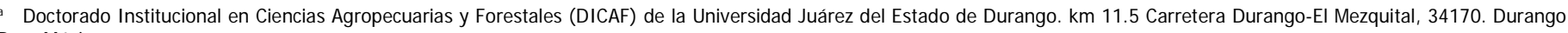
Dgo. México.

b Instituto Nacional de Investigaciones Forestales, Agrícolas y Pecuarias (INIFAP). km 4.5 Carr. Durango-El Mezquital. 34170 Durango, Dgo. México.

Facultad de Medicina Veterinaria y Zootecnia. Universidad J uárez del Estado de Durango. México.

*autor de correspondencia: jimenez.rafael@inifap.gob.mx.
} 


\section{INTRODUCCIÓN}

El sorgo dulce (Sorghum bicolor) representa una alternativa forrajera, y pueden diferenciarse variedades aptas para producción de grano y aquéllas útiles para la obtención de forraje ${ }^{(1)}$. Además, algunas variedades son consideradas de doble propósito, ya que son cultivadas para producción de forraje y obtención de grano ${ }^{(2,3)}$. En México, durante los últimos cinco años, se registró un promedio anual para la superficie sembrada con sorgo forrajero de 142 mil hectáreas y se obtuvo una producción total de 1.6 millones de toneladas de forraje verde. En este periodo, el estado de Durango aportó $13.0 \%$ de la producción nacional, con 210 mil toneladas y una superficie sembrada de alrededor de 16 mil hectáreas ${ }^{(4)}$.

En ambientes donde la disponibilidad de agua es limitada, el sorgo puede superar al maíz en rendimiento de materia seca y valor nutritivo del forraje ${ }^{(5)}$, ya que requiere cerca de $25 \%$ menos agua ${ }^{(6)}$. El conocimiento del valor nutritivo de los forrajes y concentrados alimenticios es el fundamento de la nutrición animal. Se considera que la composición química es insuficiente para caracterizar los forrajes con base en su calidad nutritiva; por ello, se recomienda el uso de otros atributos como la digestibilidad, absorción de nutrientes y metabolismo animal( ${ }^{(7)}$.

El valor nutritivo de los forrajes está directamente relacionado con su composición química y digestibilidad, las cuales son características que varían ampliamente( ${ }^{(8)}$ debido a diversos factores tales como: especie, variedad, uso de fertilizante, etapa de cosecha y condiciones ambientales registradas durante el cultivo ${ }^{(9)}$. Las pruebas de digestibilidad permiten estimar la proporción de los alimentos que puede ser degradada por el aparato digestivo(10) y el tipo de nutrientes que se tornan disponibles para el animal durante el proceso de digestión y absorción $n^{(7)}$.

La calidad del forraje debe incluir también la evaluación conjunta del contenido de proteína, digestibilidad y consumo voluntario de materia seca por parte del animal. Por ello, se recomienda evaluar el valor relativo del forraje (VRF) y la calidad relativa del forraje (CRF), los cuales son índices utilizados en la predicción y clasificación de la calidad nutricional de los forrajes mediante el análisis combinado del consumo animal esperado y la digestibilidad de la materia seca(11). Con base en los niveles obtenidos para estas variables, se considera que valores iguales y mayores a 100 denotan buena calidad nutritiva de los forrajes ${ }^{(12,13)}$. El objetivo de este trabajo fue evaluar el rendimiento y valor nutricional del forraje obtenido en tres variedades de sorgo dulce cultivadas en cuatro ambientes del estado de Durango.

\section{MATERIAL Y MÉTODOS}

El estudio se realizó en el ciclo primaveraverano de 2014, en cuatro ambientes del estado de Durango (Cuadro 1). En cada localidad se sembró en fechas diferentes debido a las condiciones meteorológicas (temperatura mínima $>10{ }^{\circ} \mathrm{C}$; Cuadro 2), recomendaciones de cultivo y disponibilidad de la parcela. La siembra se realizó entre el 3 de junio y 1 de agosto de 2014 (Cuadro 1). En cada ambiente se sembraron tres variedades de sorgo dulce, conocidas como Lico, TOM 3 y Mercedes, las cuales se liberaron por el INIFAP en 2014 $4^{(14)}$ y se desconocía su composición nutricional. La siembra de cada variedad se efectuó en parcelas de $50 \mathrm{~m}$ de longitud y $10 \mathrm{~m}$ de ancho, con una distancia entre surcos de $0.81 \mathrm{~m}$ y una profundidad de siembra de $6 \mathrm{~cm}$. El suelo de los sitios de siembra es de tipo franco (arenoso y arcilloso), con un valor

Cuadro 1. Sitios de evaluación de tres variedades de sorgo dulce cultivadas en Durango

\begin{tabular}{ccccc}
\hline Ambiente & Fecha de siembra & Fecha de cosecha & Coordenadas & Altitud $(\mathrm{m})$ \\
\hline Col. Hidalgo & $03 / 06 / 2014$ & $12 / 09 / 2014$ & $24^{\circ} 10^{\prime} 31^{\prime \prime} \mathrm{N} 104^{\circ} 33^{\prime} 20^{\prime \prime} \mathrm{O}$ & 1,867 \\
CEVAG & $20 / 06 / 2014$ & $18 / 09 / 2014$ & $23^{\circ} 59^{\prime} 25^{\prime \prime} \mathrm{N} 104^{\circ} 37^{\prime} 27^{\prime \prime} \mathrm{O}$ & 1,880 \\
La Soledad & $18 / 07 / 2014$ & $27 / 10 / 2014$ & $24^{\circ} 46^{\prime} 08^{\prime \prime} \mathrm{N} 104^{\circ} 54^{\prime} 41^{\prime \prime} \mathrm{O}$ & 1,980 \\
La Goma & $01 / 08 / 2014$ & $14 / 11 / 2014$ & $25^{\circ} 28^{\prime} 21^{\prime \prime} \mathrm{N} 103^{\circ} 42^{\prime} 23^{\prime \prime} \mathrm{O}$ & 1,162 \\
\hline
\end{tabular}


promedio de pH de 6.5. Se utilizó la dosis de fertilización recomendada para maíz forrajero (20090-00)(15), ajustada con base en los análisis previos de suelo y considerando que el ciclo biológico del sorgo es más corto en comparación con el maíz. Por ello, se utilizó la dosis media de 100-90-00, para los niveles de nitrógeno, fósforo y potasio $\left(\mathrm{N}-\mathrm{P}_{2} \mathrm{O}_{5}-\right.$ $\left.\mathrm{K}_{2} \mathrm{O}\right)$.

Además de la lluvia, ocurrida durante el periodo de estudio (Cuadro 2), se aplicó desde uno ( $\mathrm{La}$ Soledad), dos (Colonia Hidalgo) y hasta tres riegos de auxilio (La Goma), para evitar el estrés hídrico severo en las plantas. El control de la maleza se realizó de forma mecanizada, con dos pasos de escarda, y estos fueron complementados con un deshierbe manual. La cosecha se realizó a $20 \mathrm{~cm}$ de altura en la parte basal del tallo, cuando la planta estaba en la etapa fenológica de llenado del grano, lo cual se determinó presionando el grano entre los dedos, índice y pulgar, hasta que se obtuvo líquido blanco y espeso(16). Esta etapa fenológica ocurrió 101 días después de la siembra (DDS) en La Colonia Hidalgo, en CEVAG a los 91 DDS, en La Soledad a los 101 DDS y en La Goma a los 103 DDS, con un promedio general de 99 DDS.

Para la evaluación del rendimiento de forraje verde, en la parcela de cada variedad se obtuvieron cinco muestras de campo (en el esquema de cinco de oros) ${ }^{(17)}$ y las muestras consistieron de dos surcos de $5 \mathrm{~m}$ y $0.81 \mathrm{~m}$ de separación $\left(8.1 \mathrm{~m}^{2}\right)$. Las muestras de forraje se pesaron en campo con una báscula romana (Rotter ${ }^{\mathrm{TM}}$ ), de $100 \mathrm{~kg}$ de capacidad, para obtener el rendimiento de forraje verde. Se obtuvo una submuestra de $5 \mathrm{~kg}$, la cual se picó a un tamaño de partícula de $5 \mathrm{~cm}$ para su secado en bolsas de papel, que fueron introducidas a una estufa de aire forzado a $60{ }^{\circ} \mathrm{C}$ durante $72 \mathrm{~h}$. Luego del secado, las muestras se pesaron nuevamente para determinar la proporción de biomasa seca y extrapolar este valor a la muestra completa obtenida en cada parcela.

Las submuestras secas se molieron en un equipo eléctrico (Wiley ${ }^{\circledR}$ ) con criba de $1 \mathrm{~mm}$ y la harina obtenida se utilizó para determinar la composición química y la digestibilidad verdadera in vitro de la materia seca (DIVMS). La proporción de materia seca (MS) se obtuvo a partir de una muestra sometida a una temperatura de $100{ }^{\circ} \mathrm{C}$. La proporción (\%) de proteína cruda (PC) se evaluó por el método Kjeldahl, según la $A O A C^{(18)}$, el cual incluye la cuantificación del nitrógeno total y luego el valor obtenido se multiplicó por el factor 6.25. La fibra en detergente neutro (FDN), fibra en detergente ácido (FDA) y lignina (L) se determinaron con base en el método $\mathrm{ANKOM}^{(19)}$, en el cual se incluye la utilización de bolsas ANKOM F57. La celulosa se calculó por diferencia (Cel= FDA-lignina), al igual que la hemicelulosa $(\mathrm{HE}=\mathrm{FDN}-\mathrm{FDA})$.

Las muestras de sorgo se sometieron a una fermentación anaeróbica para la determinación de la digestibilidad verdadera in vitro de la materia seca (DIVMS). La fermentación se realizó con líquido ruminal, el cual se obtuvo de dos bovinos machos fistulados, con un peso de $700 \mathrm{~kg}$, los cuales se alimentaron con heno de alfalfa y concentrado comercial con $12 \%$ de proteína. El proceso de fermentación se realizó en el incubador Daisy" (ANKOM Technology Corp., Macedon, NY) siguiendo el protocolo sugerido por el fabricante ${ }^{(20)}$.

El valor relativo de forraje (VRF) y la calidad relativa de forraje (CRF) se calcularon a partir de las siguientes ecuaciones:

$V R F=\frac{(C M S)(M S D)}{1.29}$

Cuadro 2. Variables climáticas registradas en los sitios de evaluación del rendimiento y calidad del forraje en tres variedades de sorgo dulce en Durango

\begin{tabular}{ccccc}
\hline Ambiente & Temperatura máxima $\left({ }^{\circ} \mathrm{C}\right)$ & Temperatura mínima $\left({ }^{\circ} \mathrm{C}\right)$ & Lluvia acumulada $(\mathrm{mm})$ & Tipo de suelo \\
\hline Colonia Hidalgo & 28.4 & 14.3 & 333.6 & Solonetz álbico \\
CEVAG & 27.7 & 14.2 & 311.2 & Castañozem lúvico \\
La Soledad & 26.1 & 11.5 & 186.8 & Vertisol mázico \\
La Goma & 30.8 & 17.4 & 100.8 & Regosol calcárico \\
\hline
\end{tabular}


$[(0.779)(\% F D A)]$

Donde: $\quad C M S=\frac{120}{\% F D N} \quad M S D=88.9-$

$C R F=\frac{(C M S)(N D T)}{1.23}$

Donde: $C M S=\frac{120}{\% F D N} \quad N D T=M O D ; \quad M O D=$ $(M O)(D I V M O)$

$\mathrm{CMS}=$ consumo de materia seca $(\%$ del peso vivo animal), $\mathrm{MSD}=$ materia seca digestible, $\mathrm{FDN}=$ fibra en detergente neutro, FDA = fibra en detergente ácido, NDT = nutrientes digestibles totales, $\mathrm{MOD}=$ materia orgánica digestible, $\mathrm{MO}=$ materia orgánica y DIVMO= digestibilidad in vitro de la materia orgánica(11).

Los datos obtenidos se analizaron en un diseño completamente aleatorio con arreglo factorial (4 ambientes $\times 3$ variedades) y cinco repeticiones. La comparación de medias se realizó con la prueba de Tukey $(P \leq 0.05)$ utilizando el paquete estadístico SAS ${ }^{\circledR}$ Ver. 9.2.

\section{RESULTADOS Y DISCUSIÓN}

Se detectaron diferencias ( $P<0.01$ ) (Cuadro 3) entre ambientes y variedades y la interacción de estos dos factores para el rendimiento de forraje verde (FV) y forraje seco (FS). La interacción ambiente $\mathrm{x}$ variedad resultó significativa, lo cual confirmó la modificación del rendimiento de forraje obtenido por al menos una variedad y la respuesta de ésta, en relación con las otras variedades, a través de los ambientes incluidos en el estudio. En CEVAG, se obtuvo el rendimiento más alto de FV

Cuadro 3. Cuadrados medios del rendimiento de forraje en tres variedades de sorgo dulce cultivadas en cuatro ambientes del estado de Durango (t ha- ${ }^{1}$ )

\begin{tabular}{cccc}
\hline $\begin{array}{c}\text { Fuente de } \\
\text { variación }\end{array}$ & $\begin{array}{c}\text { Grados de } \\
\text { libertad }\end{array}$ & $\begin{array}{c}\text { Forraje } \\
\text { verde }\end{array}$ & $\begin{array}{c}\text { Forraje } \\
\text { seco }\end{array}$ \\
\hline Ambiente $(\mathrm{A})$ & 3 & $5290.8^{\star \star}$ & $140.5^{\star \star}$ \\
Variedad $(\mathrm{V})$ & 2 & $853.7^{\star \star}$ & $22.7^{\star \star}$ \\
$\mathrm{A} \times \mathrm{V}$ & 6 & $480.2^{\star \star}$ & $12.7^{\star \star}$ \\
Error & 48 & 57.5 & 1.5 \\
$\mathrm{CV}, \%$ & & 15.4 & 15.4 \\
\hline
\end{tabular}

$\mathrm{CV}=$ coeficiente de variación. ${ }^{*}(P<0.01)$.
(75.9 t ha-1) y FS (12.4 t ha-1) (Cuadro 4); mientras que, en La Goma se registró el valor más bajo de FV (34.2 t ha-1) y FS (5.6 t ha-1), debido principalmente a la presencia de pulgón amarillo (Melanaphis sacchari) durante la mayor parte del ciclo biológico del cultivo.

Las variedades TOM 3 (89.9 t ha $\left.^{-1}\right)$ y Lico (80.2 $\mathrm{t} \mathrm{ha}^{-1}$ ) registraron el valor más alto para el rendimiento de forraje verde, el cual se obtuvo en el sitio de mayor productividad (CEVAG). En el mismo sitio se obtuvo el más alto rendimiento de forraje seco con la variedad TOM 3 (14.7 t ha-1) y Lico (13.1 $\left.\mathrm{t} \mathrm{ha}^{-1}\right)$. Ambas variedades mostraron rendimientos estadísticamente iguales entre sí y superaron significativamente a la variedad Mercedes, la cual mostró un rendimiento inferior para FV (57.5 t ha-1) y FS (9.4 t ha-1).

Estos mayores rendimientos de forraje en las variedades TOM 3 y Lico, con respecto a la variedad

Cuadro 4. Rendimiento de forraje en tres variedades de sorgo dulce cultivadas en cuatro ambientes del estado de Durango (t ha-1)

\begin{tabular}{lll}
\hline Variedad & Forraje verde & Forraje seco \\
\hline & Colonia Hidalgo & \\
Lico & $41.5 \mathrm{a}$ & $6.8 \mathrm{a}$ \\
TOM 3 & $35.8 \mathrm{a}$ & $5.8 \mathrm{a}$ \\
Mercedes & $37.5 \mathrm{a}$ & $6.1 \mathrm{a}$ \\
Promedio & $38.3 \mathrm{C}$ & $6.2 \mathrm{C}$ \\
& CEVAG & \\
Lico & $80.2 \mathrm{a}$ & $13.1 \mathrm{a}$ \\
TOM 3 & $89.9 \mathrm{a}$ & $14.7 \mathrm{a}$ \\
Mercedes & $57.6 \mathrm{~b}$ & $9.4 \mathrm{~b}$ \\
Promedio & $75.9 \mathrm{~A}$ & $12.4 \mathrm{~A}$ \\
& La Soledad & \\
Lico & $55.3 \mathrm{a}$ & $9.0 \mathrm{a}$ \\
TOM 3 & $53.4 \mathrm{a}$ & $8.7 \mathrm{a}$ \\
Mercedes & $36.5 \mathrm{~b}$ & $6.0 \mathrm{~b}$ \\
Promedio & $48.4 \mathrm{~B}$ & $7.9 \mathrm{~B}$ \\
& La Goma & \\
Lico & $24.9 \mathrm{~b}$ & $4.1 \mathrm{~b}$ \\
TOM 3 & $40.9 \mathrm{a}$ & $6.7 \mathrm{a}$ \\
Mercedes & $36.8 \mathrm{a}$ & $6.0 \mathrm{a}$ \\
Promedio & $34.2 \mathrm{C}$ & $5.6 \mathrm{C}$ \\
\hline
\end{tabular}

Literales diferentes en la misma columna representan diferencias significativas $(P<0.01)$ entre ambientes $A-c \vee v$ variedades ${ }^{a-c}$ 
Mercedes, se debió principalmente a que esta última presentó porte bajo y precocidad a floración ( 81 días después de la siembra, DDS) (datos no mostrados)(21). La precocidad, fue una característica que permitió a la variedad Mercedes igualar estadísticamente el rendimiento de FV y FS obtenido con la variedad Lico en La Goma y a las dos variedades (Lico y TOM 3) en la Colonia Hidalgo. Dicha respuesta se debió a la acumulación temprana de materia seca en las espigas, gracias a la precocidad a floración, y la posterior continuación del crecimiento mediante el desarrollo de nuevos tallos y brotes en la parte apical del tallo. Lo anterior se observó en menor nivel en el CEVAG debido a la menor temperatura registrada en ese ambiente, en relación con La Goma y la Colonia Hidalgo. En sorgo dulce se ha observado plasticidad fenotípica para el número de tallos y las variedades con mayor cantidad de estos muestran adaptación para maximizar el uso de los recursos en ambientes favorables ${ }^{(22)}$, especialmente la temperatura ambiental y humedad del suelo.

Se detectaron diferencias $(P<0.01)$ entre ambientes, para la mayoría de las variables relacionadas con la composición química del forraje (Cuadro 5). La interacción localidad x variedad resultó no significativa, lo cual se relacionó con la similitud de la composición química de al menos una variedad entre ambientes de prueba; así como el mantenimiento de su respuesta al compararla con las otras variedades. Los valores significativamente más altos de proteína se registraron en La Goma, con un promedio de $10.0 \%$; mientras que en La Soledad se obtuvo un valor medio de $5.6 \%$ (Figura 1a). Los resultados obtenidos fueron similares a los reportados por Núñez et $a^{(23)}$, quienes obtuvieron valores de PC en un rango de 5.0 a $7.4 \%$ para variedades de sorgo cultivadas en La Laguna. El contenido de proteína fue influenciado por la respuesta de las variedades a las diferencias en la fecha de siembra, ya que se ha observado que el contenido proteico se incrementa con el retraso de las siembras ${ }^{(24)}$. Otros factores que pudieron influir fueron el manejo agronómico y la interacción de éste con la variedad y las condiciones meteorológicas de cada ambiente.

En el caso de La Goma, se observaron valores más altos y similares a los registrados en otros estudios con sorgo, en los cuales se obtuvieron proporciones de proteína entre 10.9 y $14.8 \%{ }^{(25)}$. El incremento en la proporción de proteína observado en La Goma, puede ser explicado principalmente con el retraso en la fecha de siembra ${ }^{(24)}$. Dichos factores influyeron en el crecimiento, desarrollo y el mantenimiento de una proporción alta de proteína en la planta debido al retraso en el llenado del grano, especialmente en las variedades Lico y TOM 3.

El bajo contenido de proteína que se registró en la mayoría de los sitios de evaluación se relacionó con la fecha de cosecha, ya que en el Norte de México se considera que la mejor etapa para el corte del forraje de sorgo dulce es el periodo cercano a la madurez (grano masoso-lechoso) ${ }^{(23)}$. Lo anterior, a pesar de que se ha demostrado que la mayoría de las gramíneas deben cortarse en la etapa de prefloración (embuche), para evitar problemas por lignificación y reducción del contenido de proteína ${ }^{(26)}$.

Cuadro 5. Cuadrados medios de variables evaluadas en tres variedades de sorgo dulce cultivadas en cuatro ambientes del estado de Durango

\begin{tabular}{lllllllll}
\hline Fuente de variación & $\mathrm{GL}$ & $\mathrm{PC}$ & $\mathrm{FDN}$ & $\mathrm{FDA}$ & $\mathrm{CEL}$ & $\mathrm{HE}$ & LIG & DIVMS \\
\hline Ambiente (A) & 3 & $52.0^{* *}$ & $497.4^{* *}$ & $249.7^{* *}$ & $249.5^{* *}$ & $42.5^{* *}$ & $35.3^{* *}$ & $637.0^{* *}$ \\
Variedad (V) & 2 & $3.1 \mathrm{~ns}$ & $32.3 \mathrm{~ns}$ & $14.6 \mathrm{~ns}$ & $14.8 \mathrm{~ns}$ & $5.4 \mathrm{~ns}$ & $3.6^{* *}$ & $22.5 \mathrm{~ns}$ \\
A x V & 6 & $0.9 \mathrm{~ns}$ & $24.3 \mathrm{~ns}$ & $12.9 \mathrm{~ns}$ & $13.0 \mathrm{~ns}$ & $8.1^{*}$ & $1.7^{*}$ & $27.3^{*}$ \\
Error & 48 & 2.2 & 11.1 & 5.7 & 5.7 & 3.1 & 0.6 & 9.5 \\
CV, \% & & 18.2 & 5.5 & 6.7 & 7.0 & 7.2 & 16.0 & 4.4 \\
\hline
\end{tabular}

$\mathrm{GL}=$ grados de libertad; $\mathrm{PC}=$ proteína cruda; $\mathrm{FDN}=$ fibra en detergente neutro; $\mathrm{FDA}=$ fibra en detergente ácido; $\mathrm{CEL}=$ celulosa; $\mathrm{HE}=$ hemicelulosa; $\mathrm{LIG}=$ lignina; DIVMS= digestibilidad verdadera in vitro de la materia seca; $\mathrm{CV}=$ coeficiente de variación.

$*(P<0.05), * *(P<0.01) ; \mathrm{ns}=$ no siqnificativo. 
En La Goma, se registraron niveles significativamente altos de FDN (67.7 \%), FDA (41.1\%), celulosa (39.6\%) y HE (26.3\%), en relación con el resto de los ambientes que se incluyeron en el estudio (Figuras 1 b, c, d y e). Los valores fueron ligeramente inferiores a los que se registraron en otros estudios con sorgo ${ }^{(27)}$, en los cuales el valor de FDN fue $70.7 \%$ y FDA $44.4 \%$; mientras que la proporción de celulosa resultó inferior (34.6\%). En La Soledad, se observaron los valores promedio más bajos de FDN (53.7\%), FDA (31.2\%), celulosa
(29.7\%) y lignina (3.4\%) (Figura 1f), lo cual favoreció el incremento de la DIVMS (78.2\%) (Figura 1g).

El mayor promedio de DIVMS (78.2 \%) se registró en La Soledad, Dgo. y en ese ambiente, la variedad TOM 3 fue la que presentó el valor más alto de digestibilidad, con $79.6 \%$, aunque resultó estadísticamente igual a Mercedes (78.8\%) (Figura $1 \mathrm{~g})$. Lo anterior, se relacionó con un menor contenido de fibras, puesto que en La Soledad se registraron valores significativamente más bajos de

Figura 1. Características nutricionales de tres variedades de sorgo cultivadas en cuatro ambientes diferentes del estado de Durango

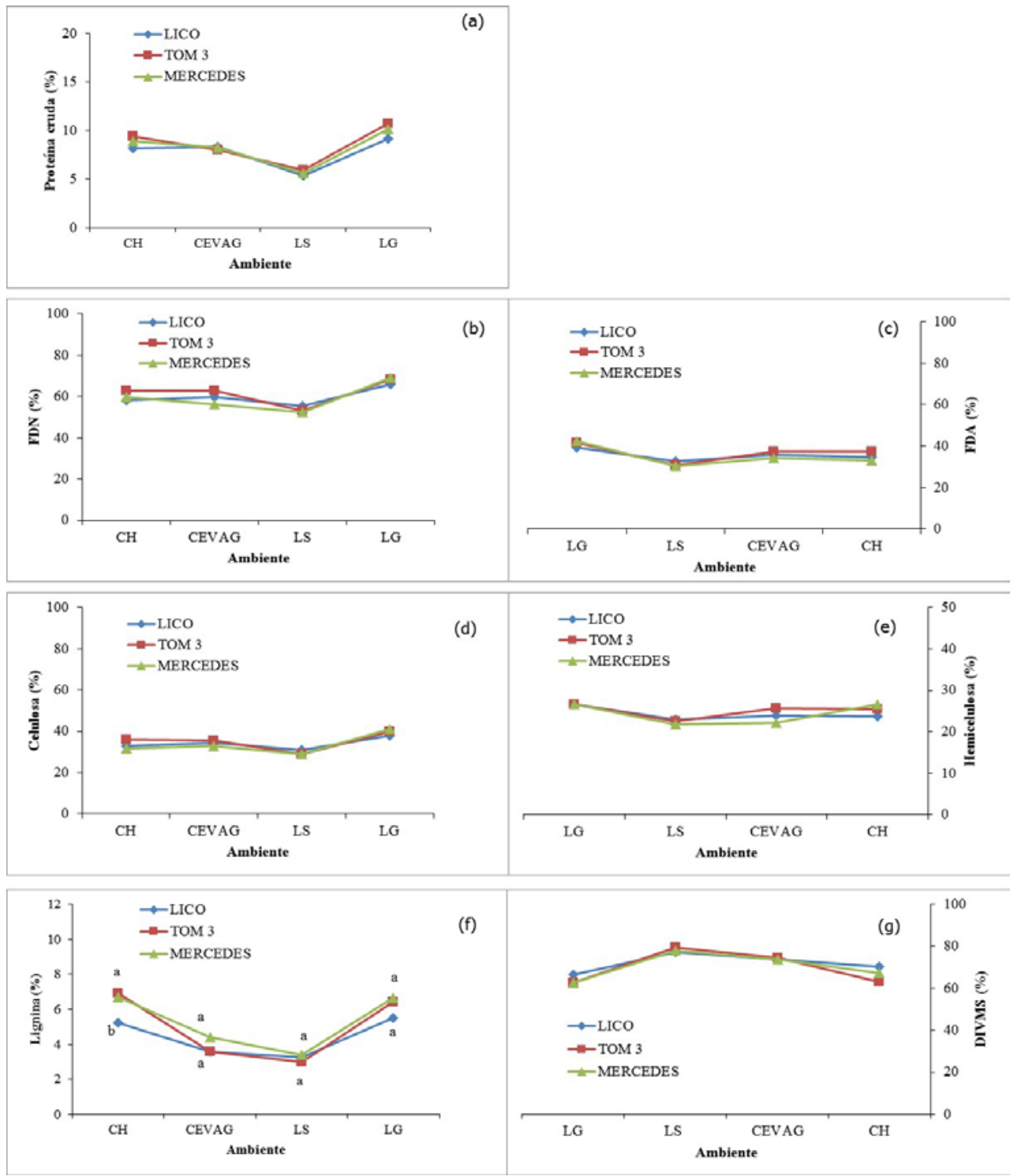


FDA (31.2 \%), FDN (53.7\%) y lignina (3.4\%) (Figura 1f).

En CEVAG, la variedad con mayor contenido de lignina fue TOM 3 (4.4\%) (Figura 1f) y a pesar de lo anterior, los datos registrados en este trabajo fueron inferiores a los reportados por Núñez et $a{ }^{(23)}$, quienes encontraron valores de lignina entre 7.4 y $9.0 \%$ en otras variedades de sorgo cultivadas en La Laguna. Las diferencias se debieron al uso de otras variedades y a la variación en el clima y tipo de suelo. Por otro lado, en La Goma, Dgo., se registró la DIVMS más baja (64.0 \%) y la lignina presentó el valor promedio más alto (6.2\%), aunque estadísticamente igual al registrado en la Colonia Hidalgo (6.3\%). Los resultados anteriores permitieron corroborar la relación negativa y significativa que existió entre la DIVMS y el contenido de lignina $\left(r=-0.86^{* *}\right)$, debido a la indigestibilidad de este compuesto presente en los forrajes ${ }^{(5)}$.

El forraje cosechado mostró valores significativamente altos $(P \leq 0.01)$ de lignina en la Colonia Hidalgo (6.3\%) y en La Goma (6.2\%) (Figura 1f). Los valores significativamente inferiores de lignina se obtuvieron con la variedad Mercedes en La Soledad (3.0\%) y CEVAG (3.2 \%). Esta variedad mostró porte bajo $(2.1 \mathrm{~m})$ y precocidad a la emergencia de la espiga (81 días después de la siembra, DDS), en comparación con TOM 3 (2.5 m y $93 \mathrm{DDS})^{(28)}$. Se ha observado, que las variedades tardías, como TOM 3 , tienen tallos más gruesos ${ }^{(29)}$ y lignificados, debido a que la lignina es la matriz de biopolímeros complejos formados en las plantas para mantener la integridad de la pared celular ${ }^{(30)}$, sostener una mayor altura de la planta(31) y soportar una mayor producción de biomasa ${ }^{(22)}$. Además, se ha observado efecto del fotoperiodo y su interacción con la temperatura ${ }^{(29)}$ en el crecimiento y desarrollo del sorgo. La variedad Mercedes mostró, en La Soledad, valores significativamente bajos de hemicelulosa (21.8\%); aunque en otros sitios se incrementó la proporción de este compuesto, como fue el caso de la Colonia Hidalgo y La Goma (26.7 \%) (Figura 1e). El balance y proporciones de los componentes químicos fluctuaron entre sitios para una misma variedad, por lo que se observó dificultad para el uso individual de estas variables en la medición de la calidad forrajera del sorgo dulce.
Se corroboró la recomendación de realizar la cosecha del sorgo en prefloración (embuche) y hasta un poco después de la emergencia de la espiga, con el fin de favorecer la calidad nutricional del forraje y evitar los problemas de acame registrados en Durango, con algunas variedades de esta especie, que muestran espigas muy altas y debilidad en tallos y raíces.

Se observaron diferencias $(P<0.01)$ entre ambientes y entre variedades $(P<0.05)$ para el valor relativo del forraje y la calidad relativa del forraje (Cuadros 6 y 7). En el caso de la CRF, se observó modificación de la respuesta de las variedades entre ambientes de siembra; así como en el nivel de CRF de al menos una variedad en comparación con las otras, lo cual causó que la interacción (ambiente $x$ variedad) fuera significativa. Con base en ambas variables (VRF y CRF), se puede decir que La Soledad resultó la mejor opción para la producción de forraje de calidad con el uso de sorgo dulce. Lo anterior, se relacionó con el retraso de la fecha de siembra ${ }^{(24)}$, en comparación con las otras localidades de mayor altitud (1,867 a $1,980 \mathrm{~m}$ ) y las bajas temperaturas registradas en este ambiente.

La Soledad, mostró los valores más altos para VRF (112.2\%) y CRF (135.0\%); mientras que, La Goma registró los más bajos $(\mathrm{VRF}=78.4 \%$ y $\mathrm{CRF}=$ $84.0 \%)$. En el caso del CRF, el valor más alto se observó en la variedad Mercedes (137.6\%) cultivada en La Soledad; aunque resultó estadísticamente igual a las variedades Lico ( $131.8 \%)$ y TOM 3 (135.5\%), cultivadas en el mismo ambiente. Los resultados demostraron que las variedades de sorgo evaluadas

Cuadro 6. Cuadrados medios de la calidad forrajera de tres variedades de sorgo dulce cultivadas en cuatro ambientes del estado de Durango

\begin{tabular}{lccc}
\hline $\begin{array}{c}\text { Fuente de } \\
\text { variación }\end{array}$ & $\begin{array}{c}\text { Grados de } \\
\text { libertad }\end{array}$ & VRF & CRF \\
\hline Ambiente (A) & 3 & $2868.1^{* *}$ & $6573.1^{* *}$ \\
Variedad (V) & 2 & $218.6^{*}$ & $376.6^{*}$ \\
A x V & 6 & $151.7 \mathrm{~ns}$ & $225.3^{*}$ \\
Error & 48 & 66.6 & 81.7 \\
CV, \% & & 8.5 & 8.2 \\
\hline
\end{tabular}

$\mathrm{VRF}=$ valor relativo del forraje; $\mathrm{CRF}=$ calidad relativa del forraje. ns= no sianificativo, ${ }^{*}(P<0.05),{ }^{* *}(P<0.01) ; C V=$ coeficiente de variación. 
Cuadro 7. Índices de calidad forrajera de tres variedades de sorgo dulce cultivadas en cuatro ambientes del estado de Durango

\begin{tabular}{lll}
\hline \multicolumn{1}{c}{ Variedad } & $\begin{array}{c}\text { Valor relativo de } \\
\text { forraje }\end{array}$ & $\begin{array}{c}\text { Calidad relativa } \\
\text { de forraje }\end{array}$ \\
\hline Lico & Colonia Hidalgo & \\
TOM 3 & $99.2 \mathrm{a}$ & $118.2 \mathrm{a}$ \\
Mercedes & $88.7 \mathrm{a}$ & $95.3 \mathrm{~b}$ \\
Promedio & $98.8 \mathrm{a}$ & $106.7 \mathrm{ab}$ \\
& $95.5 \mathrm{~B}$ & $106.7 \mathrm{~B}$ \\
Lico & CEVAG & \\
TOM 3 & $95.4 \mathrm{ab}$ & $111.5 \mathrm{ab}$ \\
Mercedes & $88.8 \mathrm{~b}$ & $106.1 \mathrm{~b}$ \\
Promedio & $104.9 \mathrm{a}$ & $120.5 \mathrm{a}$ \\
& $96.4 \mathrm{~B}$ & $112.7 \mathrm{~B}$ \\
Lico & La Soledad & \\
TOM 3 & $106.9 \mathrm{a}$ & $131.8 \mathrm{a}$ \\
Mercedes & $113.7 \mathrm{a}$ & $135.5 \mathrm{a}$ \\
Promedio & $116.0 \mathrm{a}$ & $137.6 \mathrm{a}$ \\
& $112.2 \mathrm{~A}$ & $135.0 \mathrm{~A}$ \\
Lico & La Goma & \\
TOM 3 & $82.2 \mathrm{a}$ & $88.9 \mathrm{a}$ \\
Mercedes & $77.5 \mathrm{a}$ & $81.6 \mathrm{a}$ \\
Promedio & $75.5 \mathrm{a}$ & $81.6 \mathrm{a}$ \\
\hline
\end{tabular}

Literales diferentes en la misma columna representan diferencias altamente significativas $(P<0.01)$ entre ambientes ${ }^{A-C}$ y variedades ${ }^{a-e}$.

presentaron buena calidad nutritiva en la mayoría de los ambientes (con excepción de La Goma), ya que se obtuvieron valores superiores a $100 \%{ }^{(12)}$. Con base en los valores obtenidos, el forraje de sorgo puede clasificarse en la categoría preferente (choice), en el cual se tiene un intervalo de valores entre 140 y $160^{(32)}$. En comparación con otros forrajes, en este estudio se observó similitud para el valor relativo del forraje en comparación con

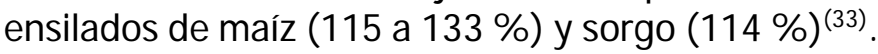

El índice de la CRF se propuso para sustituir al VRF como una medida más precisa para la predicción del comportamiento productivo animal(33), lo cual se comprobó en este trabajo. Lo anterior, debido a que el VRF subestimó la calidad de los forrajes; mientras que la CRF utilizó el total de los nutrientes digestibles e incluyó el valor de la
DIVMO(32,33), lo cual incrementó la precisión del cálculo y con ello, pudo apreciarse el valor nutritivo del forraje de sorgo dulce (Cuadro 7). A pesar de lo anterior, estas variables (VRF y CRF) mostraron eficiencia para la detección de las diferencias en la calidad forrajera observadas entre variedades y ambientes de cultivo, lo cual resultó de utilidad en la clasificación de ambientes y del germoplasma de sorgo dulce evaluado en el presente estudio.

\section{CONCLUSIONES E IMPLICACIONES}

El sorgo dulce representa una opción productiva para obtener forraje en Durango, en el ciclo primavera-verano. Las variedades TOM 3 y Lico mostraron alto rendimiento de biomasa, por lo que pueden cultivarse para incrementar la producción de forraje. La variedad Mercedes puede usarse en ambientes con baja disponibilidad hídrica, debido a su precocidad y rendimiento forrajero. Con base en los resultados obtenidos, en sitios cálidos es recomendable retrasar la siembra (al mes de julio) y adelantar la cosecha de sorgo al inicio de la etapa reproductiva (embuche), para evitar el aumento considerable del contenido de fibra que se observa en etapas fenológicas más avanzadas (llenado de grano). Se recomienda la combinación de variables agronómicas (adaptación, rendimiento de biomasa, precocidad) y nutricionales (composición química, digestibilidad y CRF) para incrementar la eficiencia productiva y la calidad de forraje de sorgo dulce producido en Durango.

\section{LITERATURA CITADA}

1. Dahlberg J. Classification and characterization of sorghum. In: Smith CW, Frederiksen RA editors. Sorghum: Origin, history, technology, and production. New York, NY, USA: John Wiley \& Sons, Inc; 2000:99-130.

2. Bean BW, Baumhardt RL, McCollum III FT, McCuistion KC. Comparison of sorghum classes for grain and forage yield and forage nutritive value. Field Crop Res 2013; (142):20-26.

3. USDA. United States Department of Agriculture-Farm Service Agency. Determining eligibility of grain sorghum varieties. Notice: LP-1834. USDA Farm Service Agency, Washington, DC. USA. 2002. http://www.fsa.usda.gov/Internet/FSA_Notice/lp_1790.pdf. Accesed July 3, 2016.

4. SIAP. Servicio de Información Agroalimentaria y PesqueraSAGARPA. 2014. http://www.siap.gob.mx/. Consultado 23 Oct, 2015. 
5. Bolaños AED, Emile J-C. Distancia entre surcos en el rendimiento y calidad de la materia seca de maíz y de sorgo. Rev Mex Cienc Pecu 2011;2(3):299-312.

6. Merrill SD, Tanaka DL, Krupinsky JM, Liebig MA, Hanson JD. Soil water depletion and recharge under ten crop species and application to the principles of dynamic cropping systems. Agron J 2007;99(4):931-938.

7. Bondi AA. Nutrición animal. Zaragoza, España: Editorial Acribia, SA; 1989.

8. Fox DG, Barry ME, Pitt RE, Roseler DK, Stone WC. Application of the Cornell net carbohydrate and protein model for cattle consuming forages. J Anim Sci 1995;73(1):267-277.

9. Singh S, Kushwaha BP, Nag SK, Mishra AK, Singh A, Anele UY. In vitro ruminal fermentation, protein and carbohydrate fractionation, methane production and prediction of twelve commonly used Indian green forages. Anim Feed Sci Technol 2012;178(1-2):2-11.

10. Church DC, Pond WG. Fundamentos de nutrición y alimentación de animales. México, DF: Editorial Limusa, SA de CV y Grupo Noriega Editores; 1994.

11. Moore JE, Undersander DJ. A proposal for replacing relative feed value with an alternative: Relative forage quality. Proc Am Forage Grassland Council. Bloomington, Minnesota, USA. 2002;(11):171175.

12. Undersander $D J$. The new relative forage quality index-concept and use. Univ Wisc Ext. Madison, WI, USA. 2003.

13. NC. North Carolina State University. Forage quality: concepts and practices. North Carolina Cooperative Extension. NC, USA. 2014.

14. SNICS. Servicio Nacional de Inspección y Certificación de Semillas. Catálogo nacional de variedades vegetales. $1^{\text {er }}$ Trimestre 2016. Tlalnepantla, Edo. de México, México. 2016.

15. Castillo RA, Peña RA, López HJ. H-376: Híbrido de maíz para producción de grano y forraje en riego en Durango. Desplegable para productores Núm. 43. INIFAP-CIRNOC-Campo Experimental Valle del Guadiana. Durango, México. 2009.

16. Rao SS, Elangovan M, Akula U, Seetharama N. Characterizing phenology and growth stages of sorghum hybrids. In: Reddy Belum VS, et al editors. Sorghum improvement in the new millennium. Patencheru, Andhra Pradesh, India; 2007:16-22.

17. Espinosa RM, Ortiz CFE, Vargas VE. Muestreo de suelos y preparación de muestras. Desplegable para Productores Núm. 23. INIFAP-CIRNE-Campo Experimental Río Bravo. Tamps., México. 2012.

18. AOAC. Association of Official Analytical Chemists. Official methods of analysis. $15^{\text {th }}$ ed. Arlington, VA, USA: Association of Official Analytical Chemists. 1990.

19. ANKOM. Acid detergent fiber in feeds. Filter bag technique (ANKOM ${ }^{200}$ ). Ankom Technology 2005; www.ankom.com/09 procedures/ADF\%20Method\%20A200.pdf (verified 8 Jan. 2008). Macedon, NY, USA: Ankom Technology Corp. Accessed Jun 28, 2016.
20. ANKOM. 2011. In vitro true digestibility using DAISY incubator. Ankom Technology 2005; http://digestibility.com/media/ documents/IVDMD 0805 D200.pdf. Macedon, NY, USA: Ankom Technology Corp. Accessed J une 28, 2016.

21. Rosales SR, Núñez MOG, Nava BCA, Acosta L, ; J iménez OR, Cuéllar REI. Rendimiento forrajero y contenido de sacarosa en variedades de sorgo cultivadas en Durango, México. Memorias Semana Internacional de Agronomía. Gómez Palacio, Durango, Méx. 2010:533-538.

22. Elikana AS, Li-Min Z, Yan X, Yu-Miao Z, Zhi-Quan L, Hai-Chun J. Sweet sorghum ideotypes: genetic improvement of the biofuel syndrome. Food and Energy Security 2015;4(3):159-177.

23. Núñez HG, Payan GJ A, Peña RA, González CF, Ruiz BO, Arzola AC. Caracterización agronómica y nutricional del forraje de variedades de especies anuales en la región norte de México. Rev Mex Cienc Pecu 2010; 1(2):85-98.

24. Mahmood A, Ullah H, Shanhzad AN, Ali H, Ahmad S, Zia-Ul-Haq M, et al. Dry matter yield and chemical composition of sorghum cultivars with varying planting density and sowing date. Sains Malaysiana 2013;42(10): 1529-1538.

25. Vargas RCF. Valor nutricional y degradabilidad ruminal de genotipos de sorgo forrajero (Sorghum sp). Agron Mes 2005; 16(2):215-223.

26. Ball DM, Collins M, Lacefield GD, Martin NP, Mertens DA, Olson KE, et al. Understanding forage quality. Park Ridge, IL, USA: American Farm Bureau Federation Publication 1-01; 2001.

27. Harinarayana G, Melkania NP, Reddy BVS, Gupta SK, Rai KN, Sateesh-Kumar P. Forage potential of sorghum and pearl millet. In: El-Beltagy A, Saxena MC editors. Sustainable development and management of drylands in the twenty-first century. Proc 7th Int Conf Develop Drylands. Aleppo, Syria. 2005:292-321.

28. Jiménez OR, Rosales SR. Rendimiento forrajero de variedades de sorgo dulce cultivadas en diferentes ambientes de Durango, México. Congreso Mundial de Ganadería Tropical. Tampico, Tamps., México. 2014:144-147.

29. Hunter EL, Anderson IC. Sweet sorghum. In: Janick J editor. Horticultural reviews. New York, USA: John Wiley \& Sons, Inc; 1997(21).

30. Duncan RR. Breeding and improvement of forage sorghums for the tropics. Adv Agron 1996; (57):161-185.

31. Trouche G, Bastianelli D, Cao-Hamadou TV, Chantereau J, Rami JF, Pot D. Exploring the variability of photoperiod-insensitive sorghum genetic panel for stem composition and related traits in temperate environments. Field Crop Res 2014; (166):72-81.

32. UGA. University of Georgia. The equation used to calculate relative forage quality in Georgia. http://www.caes.uga.edu/commodities/ fieldcrops/forages/events/SHC11/RFQ_Calc_Circ.pdf. 2015. Consultado 22 Mar, 2016.

33. KSU. Kansas State University. Relative feed value measures forage quality. Forage Facts. 2015. http://www.asi.k-state.edu/doc/ forage/fora41.pdf. Consultado 22 Mar, 2016. 